\title{
Mesoporous ZnS Thin Films Prepared by a Nanocasting Route
}

\author{
Achim Fischereder, ${ }^{\dagger, \ddagger}$ Maria Luz Martinez-Ricci, ${ }^{\perp, \#}$ Alejandro Wolosiuk, ${ }^{\perp}$ Wernfried Haas, ${ }^{\ddagger}, \S$ \\ Ferdinand Hofer, ${ }^{\S}$ Gregor Trimmel, ${ }^{\dagger, \ddagger}$ and Galo J. A. A. Soler-Illia*, ${ }^{\perp}$ \\ ${ }^{\dagger}$ Institute for Chemistry and Technology of Materials, Graz University of Technology, Stremayrgasse 9, 8010 Graz, Austria \\ ${ }^{\ddagger}$ Christian Doppler Laboratory for Nanocomposite Solar Cells, Graz University of Technology and NanoTecCenter Weiz \\ Forschungsgesellschaft GmbH, Austria \\ ${ }^{\S}$ Institute for Electron Microscopy and Fine Structure Research, Graz University of Technology, Steyrergasse 17, 8010 Graz, Austria \\ ${ }^{\perp}$ Gerencia Química, Centro Atómico Constituyentes Comisión Nacional de Energía Atómica, Avenida Gral Paz 1499, B1650KNA \\ San Martín, Argentina \\ \#DQIAQF, Universidad de Buenos Aires, Facultad de Ciencias Exactas y Naturales, Ciudad Universitaria Pab. II, C1428EHA Buenos \\ Aires, Argentina
}

\section{Supporting Information}

ABSTRACT: Mesoporous metal chalcogenides (e.g., ZnS, CuS, and derived mixed sulfide or selenides) show an extremely high potential in technological areas like catalysis, sensors, environmental protection, and photovoltaics. Although chemical bath deposition methods allow obtaining stable and porous metal chalcogenide films under mild and simple conditions, the reproducible preparation of highly ordered mesoporous sulfide thin films has been challenging so far. Herein, we present a simple and efficient synthetic method to prepare $\mathrm{ZnS}$ mesoporous thin films using a combination of a nanocasting approach and a successive ionic layer adsorption and reaction (SILAR) process for the infiltration. A mesoporous silica thin film, prepared by evaporation induced self-assembly (EISA), serves as the hard exotemplate for the formation of the mesostructured zinc sulfide. After selective etching of the silica framework, a stable and porous $\mathrm{ZnS}$ thin film is obtained, which replicates the pore structure of the hard template. A thorough combination of characterization techniques is used to assess the exotemplating process as well as the template removal.

KEYWORDS: zinc sulfide, mesoporous film, nanocasting, SILAR

\section{INTRODUCTION}

Since the first reports concerning the preparation of threedimensional ordered silica networks by supramolecular templating and coassembly, mesoporous materials have attracted a lot of scientific and industrial attention. ${ }^{1,2}$ Because of their unique properties (monodisperse mesoporosity, highly ordered structure, accessible high surface area $<2000 \mathrm{~m}^{2} \mathrm{~g}^{-1}$ ), the possibility to tailor their morphological parameters such as pore size and geometry and to functionalize the mesoporous materials, these materials find prospective applications in fields such as biomedicine, catalysis, environmental protection, optics, sensors or photovoltaics. ${ }^{3-5}$ In addition to siliceous materials, mesoporous transition metal oxides, metals, carbons or metal chalcogenides can be prepared by combining the sol-gel process with surfactant templating. ${ }^{6-8}$ However, in the case of nonsiliceous materials, the chemistry involved in the controlled building of the inorganic framework (solvolysis, condensation and/or redox reactions) is much more varied and the control is not straightforward. ${ }^{9}$ In the particular case of metal sulfides, the necessity of favorable interactions between the surfactant template and the final mesoporous nanocrystalline material requires special attention. Sulfide ions must be generated in situ or slowly introduced as $\mathrm{H}_{2} \mathrm{~S}$ for a controlled precipitation of the metal sulfide within the templating structure. ${ }^{10-12}$ Simultaneously, the competition between homogeneous and hetero- geneous nucleation of semiconductor clusters must be thoroughly controlled in order to achieve the homogeneous formation of a semiconductor film, or its anchoring to colloidal surfaces. ${ }^{13}$ In addition, the removal of the surfactant soft template without destroying the mesoporous structure turns out to be difficult, as the single nanoparticles are normally weakly bound to each other, ${ }^{14}$ and oxidation reactions or fast, extended oxide crystallization might take place. Recently, high loading of transition metals salts in dual surfactant lyotropic liquid-crystalline phases allowed for synthesis of spongy mesostructured $\mathrm{CdS} / \mathrm{Se}$ and $\mathrm{ZnS} / \mathrm{Se}^{15,16}$ films with thicknesses in the range of 400-600 $\mathrm{nm}$. However, temperature must be carefully controlled in order to prevent both the lyotropic liquid crystalline phase destabilization and salt crystallization within the mesophases.

These synthetic issues can be easily solved using a nanocasting approach, in which mesoporous silica serves as a hard exotemplate. ${ }^{17-20}$ This robust and universal synthetic method allows for the preparation of novel mesoporous materials (e.g., carbons, metals, nonoxidic phases), which cannot be prepared through sol-gel processes in a straightfor-

Received: February 4, 2012

Revised: April 25, 2012

Published: April 26, 2012 
Scheme 1. Schematic Illustration of the Single Steps during the Infiltration of the MSF Substrate Using SILAR

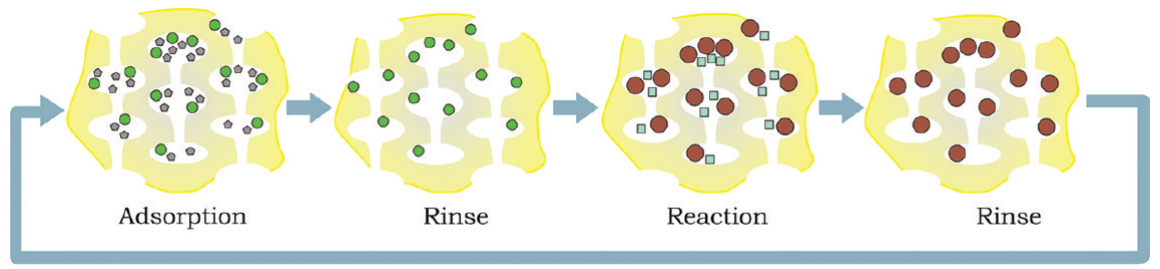

$\circ \mathrm{Zn}^{2+} \quad \mathrm{Cl}^{-} \quad \square \mathrm{Na}^{+} \quad \mathrm{ZnS}$

ward way. ${ }^{21,22}$ After infiltration of the desired material or precursor within the nanospace provided by the pores of the hard silica template, further treatments can be carried out without the danger of destroying the meso-organized structure. Therefore, even high surface area inorganic compounds, which require high temperatures for their synthesis, can be obtained. ${ }^{23}$ In addition, the interconnected three-dimensional pore structure of the mesoporous silica allows the formation of a continuous solid phase of the infiltrated nanomaterial. The selective removal of the silica template results in a stable nanostructured material, which is an inverse replica of the exotemplate. $^{24}$

Several applications in catalysis, sensing, or solar cells require processing of nonoxide materials as mesoporous thin films (MTF). Indeed, a number of promising film applications for mesoporous metal chalcogenide coatings have been described in the sensing area; for example, pressed powders obtained from nanocasting have been used. Although the nanocasting method is well-established for bulk samples (i.e., monoliths or powders), it has been less developed for MTF. ${ }^{21,22,25}$ In this context, the use of mesoporous chalcogenide thin films (i.e., $\mathrm{ZnS}$, CdS, ZnSe, CIGS, etc.) for photovoltaic applications represent an interesting approach for the design of inorganicorganic hybrid solar cells. The high surface area of a mesoporous-based material would allow controlling the charge separation while keeping an efficient charge collection based on the connectivity to the underlying electrode. On the other hand, a direct film processing pathway, compatible with the electronics industry, is a desired feature for real devices, in order to minimize steps and keep reproducible, tailored porosity. In addition to the intrinsic MTF chemical stability, film-substrate adhesion, and the preservation of the substrate properties (i.e., conductivity, transparency, texture) are essential issues if optoelectronics applications in solar cells or sensors are sought for.

In this contribution, we focus on the synthesis and characterization of mesoporous $\mathrm{ZnS}$ thin films using a nanocasting approach. Mesoporous silica thin films prepared by an evaporation induced self-assembly method (EISA) are used as a hard template, ${ }^{26}$ which is infiltrated, in a subsequent step, with $\mathrm{ZnS}$ by a successive ionic layer adsorption and reaction (SILAR) process. ${ }^{27-29}$ The silica exotemplate is then selectively etched away to expose the stable mesoporous $\mathrm{ZnS}$ thin film. The steps of the preparation process as well as the final $\mathrm{ZnS}$ thin film are characterized using UV/vis, environmental ellipsometry porosimetry (EEP), scanning electron microscopy (SEM) including energy-dispersive X-ray spectroscopy (EDS) and transmission electron microscopy (TEM, bright field imaging and selected area electron diffraction, SAED). In addition, small-angle X-ray scattering (SAXS) and $\mathrm{X}$-ray reflectometry (XRR) measurements were performed.

\section{EXPERIMENTAL SECTION}

Chemicals. Tetraethoxysilane (TEOS, 99.999\%, trace metal basis), triblock copolymer Pluronics F-127 (F127, $\mathrm{PEO}_{106}-\mathrm{PPO}_{70}-\mathrm{PEO}_{106}$, $M_{\mathrm{W}} \approx 13800$ ), zinc(II) chloride ( $\mathrm{ZnCl}_{2}$, puriss. p. a.), sodium sulfide $\left(\mathrm{Na}_{2} \mathrm{~S}, 98 \%\right)$, hydrochloric acid ( $\mathrm{HCl}, 37 \%$, ACS reagent), and ethanol (EtOH, absolute) were purchased from Sigma Aldrich and used without further purification. Millipore water was used for the preparation of all precursor solutions.

Glass substrates were purchased from Marienfield (Germany) and indium tin oxide- (ITO)-coated glass substrates with a surface resistivity of $15-25 \Omega / \mathrm{cm}^{2}$ were purchased from Delta Technologies Ltd. (USA).

Preparation of Mesoporous $\mathrm{SiO}_{2}$ Thin Films. Mesoporous silica films (MSF) were prepared on ITO-covered glass (glass/ITO) substrates by a sol-gel method based on the oxide precursor tetraethoxysilane (TEOS) using Pluronic F-127 as templating agent. Samples were also deposited onto thin glass cover slips presenting a predeposited $80 \mathrm{~nm}$ thick $\mathrm{ZrO}_{2}$ buffer layer (glass $/ \mathrm{ZrO}_{2}$ ); this buffer layer was prepared by dip-coating from a $\mathrm{ZrCl}_{4} / \mathrm{EtOH} /$ water solution of molar composition 1:40:20, and subsequent firing to $350{ }^{\circ} \mathrm{C}$. Prior to deposition, the substrates were washed with Dextran or other suitable surfactant-based cleaning agents, followed by rinsing with water and ethanol. Precursor films were prepared by dip-coating with a withdrawing speed of $4 \mathrm{~mm} \mathrm{~s}^{-1}$ under $40-50 \%$ relative humidity $(\mathrm{RH})$ at room temperature. The precursor solution was prepared using the following molar ratios: 1 TEOS: 0.0055 F127: 40 EtOH: $10 \mathrm{H}_{2} \mathrm{O}$ : $0.009 \mathrm{HCl}$. Deposited films were stored immediately in a chamber with $50 \% \mathrm{RH}$ for $30 \mathrm{~min}$ followed by a stabilizing thermal treatment of $30 \mathrm{~min}$ at $60{ }^{\circ} \mathrm{C}$ and $30 \mathrm{~min}$ at $130{ }^{\circ} \mathrm{C}$. The final calcination was carried out at $350{ }^{\circ} \mathrm{C}$ for $2 \mathrm{~h}$. After this treatment, robust and adhesive $\mathrm{SiO}_{2}$ films with a well-defined mesoporous structure were obtained.

Infiltration of the Mesoporous $\mathrm{SiO}_{2}$ Films. To infiltrate the mesoporous $\mathrm{SiO}_{2}$ films with $\mathrm{ZnS}$ (MSF: $\mathrm{ZnS}$ ), we used a successive ionic layer adsorption and reaction (SILAR) process. Each infiltration cycle consisted of four steps as represented in Scheme 1: in the first step the $\mathrm{SiO}_{2}$ film is placed for $1 \mathrm{~min}$ in a $0.1 \mathrm{M}$ aqueous solution of $\mathrm{ZnCl}_{2} . \mathrm{Zn}(\mathrm{II})$ species are adsorbed on the silica surface, forming an electrical double layer with the $\mathrm{Cl}^{-}$anions. The surface adsorption of the $\mathrm{Zn}^{2+}$ ions takes place as long as the isoelectric point (i.e.p.) of the substrate (iep of $\mathrm{SiO}_{2} \approx 1.7-3.5$ ) is below the $\mathrm{pH}$ of the $\mathrm{ZnCl}_{2}$ solution ( $\mathrm{pH}$ 6.0). In the second step, the substrate is rinsed with distilled water to remove unadsorbed $\mathrm{Zn}^{2+}$ and $\mathrm{Cl}^{-}$ions from the diffuse layer. In the third step the substrate is immersed into a $0.07 \mathrm{M}$ aq. $\mathrm{Na}_{2} \mathrm{~S}$ solution for 1 min leading to the replacement of the $\mathrm{Cl}^{-}$ions with $\mathrm{S}^{2-}$ ions. Hence, the reaction between the $\mathrm{Zn}^{2+}$ and $\mathrm{S}^{2-}$ ions occurs, leading to the formation of insoluble $\mathrm{ZnS}$ clusters. In the last step, the substrate is washed again with $\mathrm{H}_{2} \mathrm{O}$ to remove $\mathrm{Na}_{2} \mathrm{~S}$ in excess. This cycle was repeated several times, in order to obtain higher pore loading with $\mathrm{ZnS}$.

Characterization. UV-vis optical characterization of the films was performed with a Hewlett-Packard 8453 spectrophotometer. Environmental Ellipsometric Porosimetry (EEP) measurements were performed on a UV-visible $\left(190-1000 \mathrm{~nm}, \mathrm{MgF}_{2}\right.$ optics) variable angle spectroscopic ellipsometer (VASE) from SOPRA (GES5A), and analyzed with the WVase 32 software. Dynamic in situ monitoring of the film parameters (index of refraction and thickness) was realized by varying of the relative vapor pressures between $0 \%$ and $100 \%$ inside 
the analysis chamber. ${ }^{20}$ Field emission-scanning electron microscopy (FE-SEM) images were taken with a ZEISS LEO 982 GEMINI field emission electron microscope in the secondary-electron mode, using an in-lens detector to improve resolution. Energy-dispersive X-ray (EDS) spectra were recorded with a scanning electron microscope using a Philips SEM 515 equipped with a Falcon detector. Transmission electron microscopy (TEM) bright-field images were acquired on a Tecnai 20 microscope (FEI Company, $200 \mathrm{kV}, \mathrm{LaB}_{6}$ cathode) and selected area electron diffraction (SAED) patterns were acquired on a Tecnai 12 microscope (FEI Company, $120 \mathrm{kV}, \mathrm{LaB}_{6}$ cathode).

Film density was obtained from the analysis of the critical angle of X-ray Reflectometry (XRR) measurements that were performed at the D10A-XRD2 line of Laboratorio Nacional de Luz Sincrotron (LNLS), Campinas, SP, Brazil $(\lambda=1.5498 \AA) .{ }^{30}$ To obtain accurate density values, we completed measurements under dry nitrogen flux to avoid atmospheric water condensation within the pores which would lead to an underestimation of mesoporosity. The film mesostructure was analyzed using SAXS in transmission mode at the D11A-SAXS2 line of LNLS $(\lambda=1.608 \AA)$ at normal and $3^{\circ}$ incidence on thin glass covers.

\section{RESULTS AND DISCUSSION}

a. Characterization of MSF Exotemplate on ITO/Z $\mathrm{ZO}_{2}$ Subtrates. Mesoporous silica film (MSF) exotemplates were prepared by an evaporation induced self-assembly (EISA) process using $\mathrm{F} 127$ as templating agent. ${ }^{26}$ The preparation method involves dip-coating of substrates (glass/ITO or glass/ $\mathrm{ZrO}_{2}$ ) into the silica-based precursor solution described above under controlled atmosphere. After thermal stabilization treatment up to $350{ }^{\circ} \mathrm{C}$, robust, transparent, and well-defined mesoporous silica films on ITO or glass were obtained.

Figure 1a shows the SEM image of the top layer of an MSF prepared on an ITO-covered glass substrate. The porous
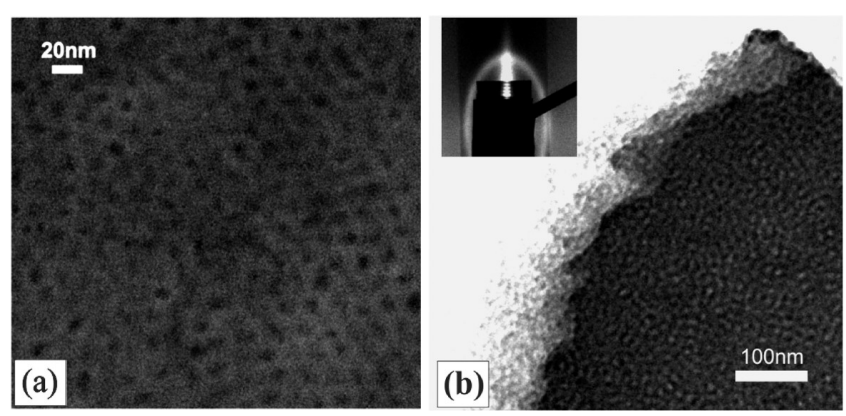

Figure 1. (a) SEM and (b) TEM images of a MSF patterned with F127. Inset in b shows a SAXS image at $3^{\circ}$ incidence.

structure can be recognized with pore sizes of approximately 6 $\mathrm{nm}$ radius measured from the SEM image. Figure $1 \mathrm{~b}$ shows a typical TEM image of the MSF, where it is possible to observe homogeneous mesopores with local order structure. Along the silica samples, regions presenting this kind of local pore order and $\operatorname{Im} 3 \bar{m}$ structure are observed. The inset in Figure $1 \mathrm{~b}$ corresponds to a 2D SAXS image of a MSF thin film over glass/ $\mathrm{ZrO}_{2}$, where the scattering pattern shows a continuous ellipsoidal figure, confirming the local order structure observed in the TEM image. ${ }^{31}$
Employing UV-VIS spectroscopic ellipsometry measurements in the range of 250 to $850 \mathrm{~nm}$, it was possible to obtain the thickness and the refractive index of the MSF on glass/ITO. For this characterization, the Cauchy model for the index of refraction is suitable for both semiconductive layers (ITO and MSF) and the glass substrate. ${ }^{32,33}$ This model expresses the functional dependence of the real and imaginary parts of the index of refraction with wavelength $n(\lambda)=n_{\mathrm{r}}(\lambda)+i n_{i}(\lambda)$, where the real part is expressed as

$$
n_{\mathrm{r}}(\lambda)=A+\frac{B}{\lambda^{2}}+\frac{C}{\lambda^{4}}
$$

and the imaginary component as

$$
n_{i}(\lambda)=\frac{D}{\lambda}+\frac{E}{\lambda^{3}}+\frac{F}{\lambda^{5}}
$$

$A, B, C, D, E$, and $F$ are parameters, whose value was adjusted according to the modeled material. In particular, in the case of transparent materials, $n_{i}(\lambda)=0$ in the wavelength range studied.

Implementing this model to each layer of the system under study (MSF and ITO), the parameters obtained, using spectroscopic ellipsometry at $0 \% \mathrm{RH}$, are specified in Table 1. The first column refers to the thickness $d$ of each layer. Refractive indexes for both films, obtained from the best fit of the ellipsometric parameters $\Psi$ and $\Delta$ to the Cauchy parameters shown in Table 1 are plotted in Figure 2.

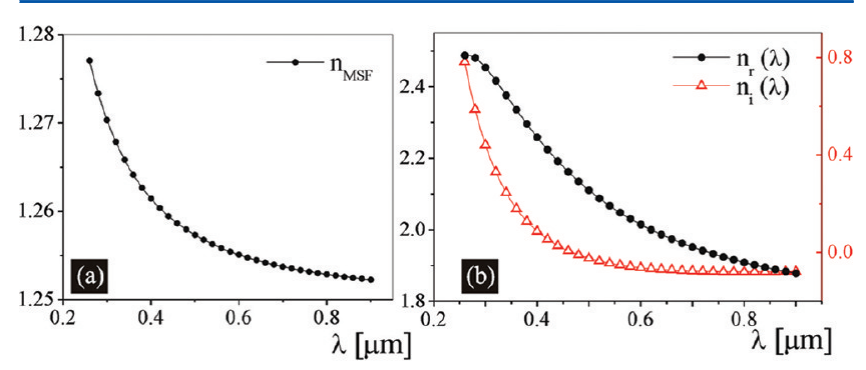

Figure 2. Refractive index curves corresponding to the parameters shown in Table 1: (a) MSF, (b) ITO layer (black circles and left axis correspond to $n_{\mathrm{r}}(\lambda)$, red triangles and right axis correspond to $n_{i}(\lambda)$ ).

The Cauchy parameters for the mesoporous silica film show, as expected, that the MSF has no absorption (i.e., $n_{i}(\lambda)=0$ ) and only two parameters are needed to properly fit the refractive index. This is not the case of the ITO layer, where six Cauchy parameters are necessary to get an appropriate index of refraction model. The last column of Table 1 shows typical refractive index values for both MSF and ITO layers at $630 \mathrm{~nm}$.

The refractive index of MSF layers changes in the presence of vapors, due to the adsorption and condensation of molecules from the gas phase into the mesopores. The EEP technique uses this phenomenon to assess morphological parameters of the pore system, such as pore volume and pore and neck size distribution. $^{34}$ Due to pore filling with water, the MSF refractive index increases from 1.26 at lower vapor pressures to 1.43 at saturation. Using an effective medium model, and the

Table 1. Thickness and Optical Parameters of the Cauchy Model for the MSF and the ITO Layers for $250 \mathrm{~nm}<\lambda<850 \mathrm{~nm}$

$\begin{array}{lcccccccc} & d(\mathrm{~nm}) & A & B\left(\mu \mathrm{m}^{2}\right) & C\left(\mu \mathrm{m}^{4}\right) & D(\mu \mathrm{m}) & E\left(\mu \mathrm{m}^{3}\right) & F\left(\mu \mathrm{m}^{5}\right) & n_{630} \\ \text { MSF } & 202.9 & 1.25 & 1.83 \times 10^{-3} & & & & 1.26+0 i \\ \text { ITO } & 159.5 & 1.76 & 0.103 & -3.62 \times 10^{-3} & -9.67 \times 10^{-2} & 2.15 \times 10^{-2} & -8.89 \times 10^{-5} & 1.99-4.31 \times 10^{-2} i\end{array}$



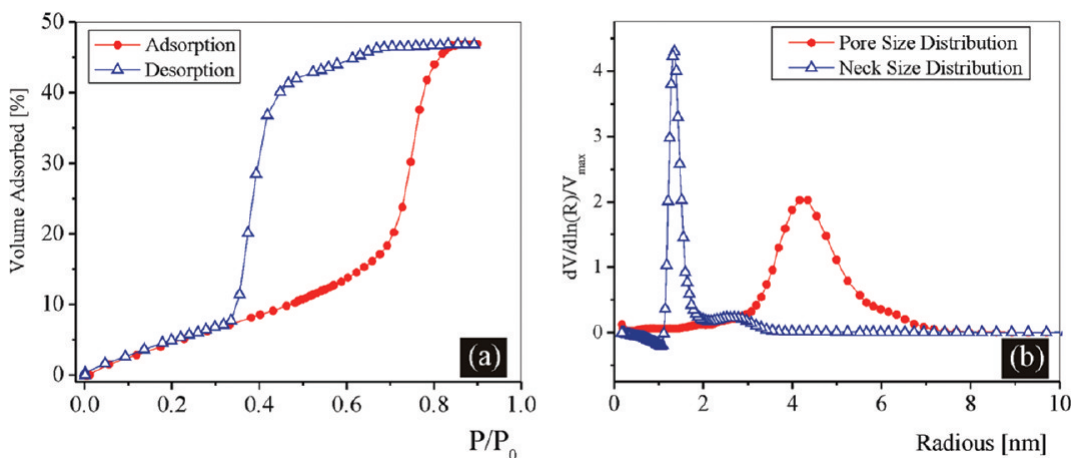

Figure 3. (a) Adsorption-desorption isotherms and (b) pore and neck size distribution of a mesoporous $\mathrm{SiO}_{2}$ thin film prepared on glass/ITO with F127 as surfactant.
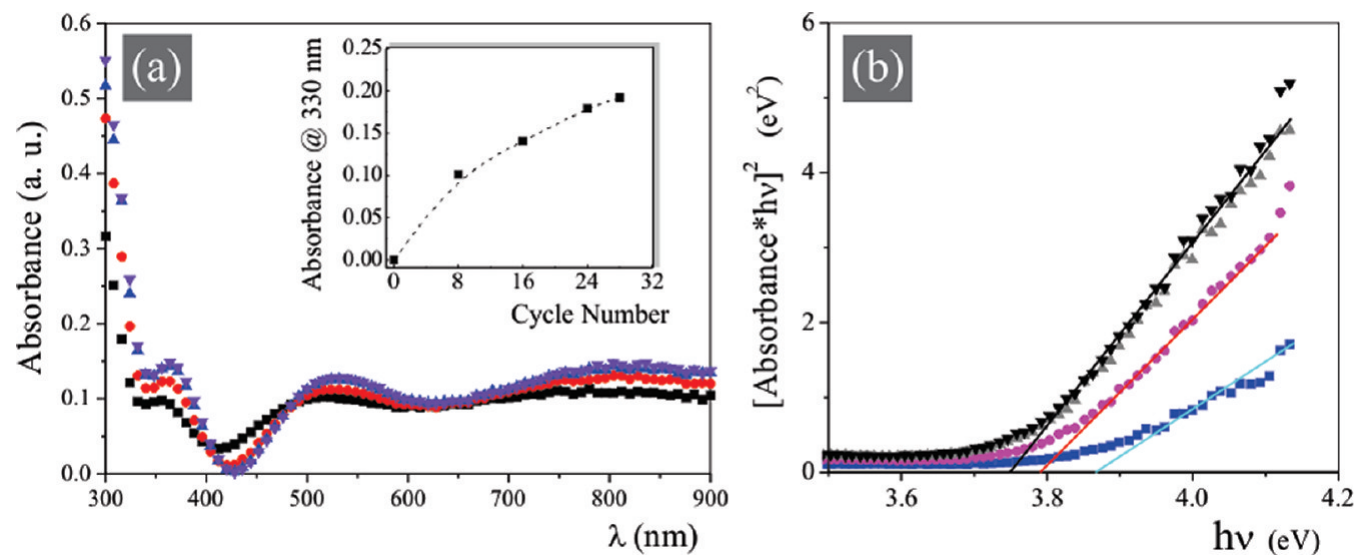

Figure 4. (a) Evolution of the absorption behavior of a MSF on glass/ITO during infiltration with ZnS. Inset: increase of absorption at $330 \mathrm{~nm}$ vs number of infiltration cycles (b) Tauc plot (i.e., $(\varepsilon h \nu)^{2}$ versus $E$ of the incident light) of ZnS-MSF nanocomposites with variable sulphide loading ( 8 cycles, 16 cycles, $\boldsymbol{\Delta} 24$ cycles, $\nabla 26$ cycles); the linear fits at high energy permit to estimate the band gap for each sample.

values of refractive index of Table 1 as seeds, the adsorbed water volume can be calculated for all the range of vapor pressures. Figure $3 \mathrm{a}$ shows the adsorption-desorption isotherms obtained by applying this model to an MSF prepared on an ITO-covered glass substrate. The isotherms present Type IV behavior, showing a steep increase/decrease in adsorption/ desorption due to capillary condensation, and $\mathrm{H} 2$ hysteresis loops, characteristic of cage-like mesopores with restrictions. ${ }^{35}$ Figure 3a shows that the overall accessible porosity of the film is approximately $47 \%$. The pore and neck size distribution can be calculated from the adsorption and desorption isotherms respectively. In mesoporous samples presenting $\mathrm{H} 2$ loops, pore sizes are obtained from adsorption the isotherm that reflects equilibrium, and the neck diameter can be obtained from the desorption curves. ${ }^{36}$ The film exhibits pore and neck radii of around 4.5 and $1.3 \mathrm{~nm}$ respectively, based on the Kelvin equation $^{37,38}$ (Figure $3 b$ ), which is in excellent agreement with literature data ${ }^{39}$ and the results obtained from the SEM image analysis (Figure 1).

b. ZnS Infiltration: Characterization of MSF:ZnS Nanocomposite Films. $\mathrm{ZnS}$ is a wide gap semiconductor with multiple applications such as light emitting diodes, IR windows, transparent conductive coatings, and solar cell buffer layers. ${ }^{40}$ Controlled synthesis of $\mathrm{ZnS}$ within the pore system of the MSF exotemplate was performed using the SILAR process described in the Experimental Section and illustrated in Scheme 1. Alternate immersion of the film in solutions of a metal salt,
$\mathrm{ZnCl}_{2}$, and a sulfur source, $\mathrm{Na}_{2} \mathrm{~S}$ is used to grow the semiconductor crystals in subnanometer steps. ${ }^{19,41}$

This simple approach allows a controlled $\mathrm{ZnS}$ infiltration within the $\mathrm{SiO}_{2}$ exotemplate although there is a competition between this process and the nucleation of $\mathrm{ZnS}$ clusters on the external surface of the MSF. Nonetheless, this issue can be minimized working with dilute $\mathrm{ZnS}$ precursor solutions, as described in this work.

$\mathrm{UV} / \mathrm{vis}$ spectroscopy was employed in order to track the MSF infiltration with $\mathrm{ZnS}$ in each SILAR cycle, as shown in Figure 4a. A significant increase of the absorption below 340 $\mathrm{nm}$ is observed due to the formation of $\mathrm{ZnS}$ (typical band gap $\mathrm{ZnS} 3.6-3.8 \mathrm{eV}$ ). The shift in the absorption fringes appears because the total optical path changes at each $\mathrm{ZnS}$ infiltration step due to the variation in the refractive index of the film matrix as the $\mathrm{ZnS}$ fills the mesopores.

The band gap of MSF:ZnS composites with different $\mathrm{ZnS}$ loading was obtained by plotting the data as the well-known Tauc plot, i.e., $(\varepsilon \mathrm{h} \nu)^{2}$ versus the energy of the incident light in $\mathrm{eV}, h \nu$. After extrapolation of the linear region to zero, a steady decrease in the $E_{\mathrm{g}}$ from 3.86 to $3.75 \mathrm{eV}$ is observed as infiltration proceeds. ${ }^{42}$ These values are well above the $E_{\mathrm{g}}$ bulk values for cubic $\mathrm{ZnS}, 3.6 \mathrm{eV}$ and hexagonal $\mathrm{ZnS}, 3.8 \mathrm{eV}^{43}$ Independently of the $\mathrm{ZnS}$ crystalline phase present in the nanocomposites, it is clear that as the $\mathrm{ZnS}$ nanoparticles grow with each infiltration step, the observed $E_{\mathrm{g}}$ converges to the bulk value. Interestingly, after 24 SILAR cycles, the optical 
absorption seems to reach a plateau (inset in Figure 4a), suggesting that $\mathrm{ZnS}$ infiltration reaches an upper limit.

The infiltration process is clearly observed by electron microscopy. Comparing SEM micrographs along the MSF films before and after 28 cycles of infiltration (Figure 5), it is evident

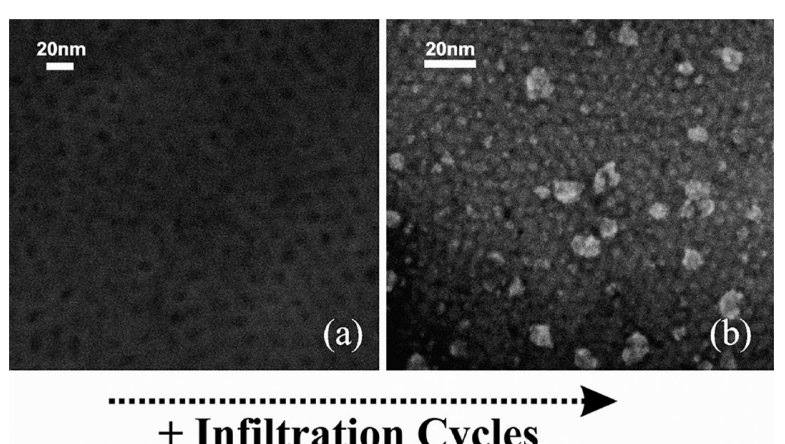

+ Infiltration Cycles
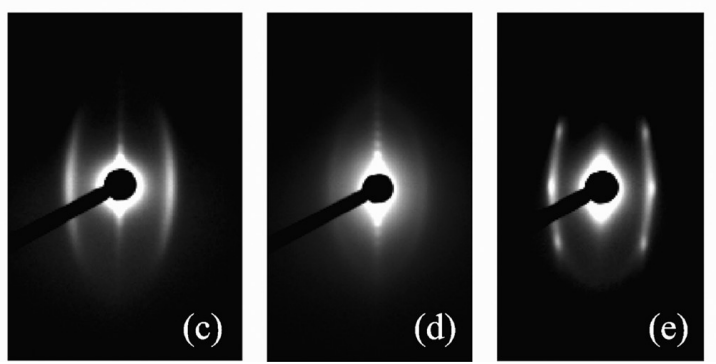

Figure 5. SEM images of a MSF prepared on glass/ITO (a) before infiltration and (b) after 28 cycles of infiltration. SAXS images of a MSF on glass $/ \mathrm{ZrO}_{2}$ for: (c) 0 , (d) 14, and (e) 28 cycles.

that, at least, surface pores or walls are filled with $\mathrm{ZnS}$. In particular, $\mathrm{SiO}_{2}$ pores that appear as dark spots in Figure 5a turn white as they are filled with $\mathrm{ZnS}$, as shown in Figure $5 \mathrm{~b}$. This change in the image intensity is caused by the fact that heavy elements (e.g., $\mathrm{Zn}$ ) produce more secondary electrons than lighter elements (e.g., Si). Therefore, in SEM images, regions containing heavier elements appear brighter than regions with lighter elements. This result demonstrates that the SILAR process leads to a uniform infiltration of the mesoporous structure with $\mathrm{ZnS}$.

The same qualitative trend is observed when analyzing SAXS patterns of empty and infiltrated MSF. Mesoporous silica samples on ITO display continuous ellipsoidal diffraction patterns corresponding to a mixture of an $\operatorname{Im} 3 m$ and a local order structure (Figure 5c). After $14 \mathrm{ZnS}$ infiltration cycles, the diffraction signal decreases (Figure 5d); after $28 \mathrm{ZnS}$ infiltration cycles, a strong pattern typical of a highly ordered cubic $\operatorname{Im} 3 \mathrm{~m}$ mesostructure is observed (Figure 5e). These results can be interpreted by taking into account the changes in the periodic electron density of the nanocomposites before and after infiltration. The decrease in scattering intensity observed for the first infiltration cycles can be explained in the basis of the decrease of electron density contrast between the MSF walls and the pores that take place upon partial pore filling. Scattering intensity can be lost upon partial loading due to the inhomogeneous distribution of the $\mathrm{ZnS}$ particles on the pore walls along the initial filling steps. In consequence, the initial SAXS pattern disappears but the original MSF mesostructure does not suffer any structural change as seen in the SEM images. When a higher loading of MSF with $\mathrm{ZnS}$ is achieved, the appearance of a cubic structure SAXS pattern is observed, due to the reappearing coherence in the periodic electron density structure. This fact is in excellent agreement with Figure $5 b$ where a great fraction of the $\mathrm{SiO}_{2}$ surface pores appear homogeneously filled with $\mathrm{ZnS}$. In a previous work on $\mathrm{Ag}$ infiltration on mesoporous oxide matrices, the same SAXS signal disappearance was observed along pore filling with metallic nanoparticles. ${ }^{40}$ In that case, the $\mathrm{Ag}$ nanoparticles were deposited following a random distribution along the mesoporous structure which in turn, disrupted the electronic periodic density. In this case, $\mathrm{ZnS}$ clusters grow homogeneously within the $\mathrm{SiO}_{2}$ pores in a step by step fashion, ultimately leading to an inverse structure with a characteristic SAXS signal that keeps the symmetry of the mesopore system. It can be proposed that a small nanoparticle filling will blur the electronic density contrast; however, at higher loadings, the sulfide being significantly denser than silica (see below), a higher electronic contrast will result, and therefore, the X-ray scattering signal will be higher. A quantitative assessment of the $\mathrm{ZnS}$ loading is needed in order to understand this issue.

For a rigorous quantification of the $\mathrm{ZnS}$ loadings in the MSF, XRR measurements following previously published procedures were performed. ${ }^{40}$ XRR signals depend on the electron density of the thin film, following the expression

$$
\rho_{\mathrm{el}}=\frac{\pi}{\lambda^{2} r_{\mathrm{e}}} \theta_{\mathrm{c}}^{2}
$$

where $\lambda$ is the $\mathrm{X}$-ray wavelength, $r_{\mathrm{e}}$ is the classical electron radius $\left(2.813 \times 10^{-6} \mathrm{~nm}\right)$, and $\theta_{\mathrm{c}}$ is the critical angle at which total reflection of the $\mathrm{X}$-ray takes place. The critical angle depends on the electron density of the film, and is distinguished as an abrupt decay in the XRR signal. Figure 6a shows a
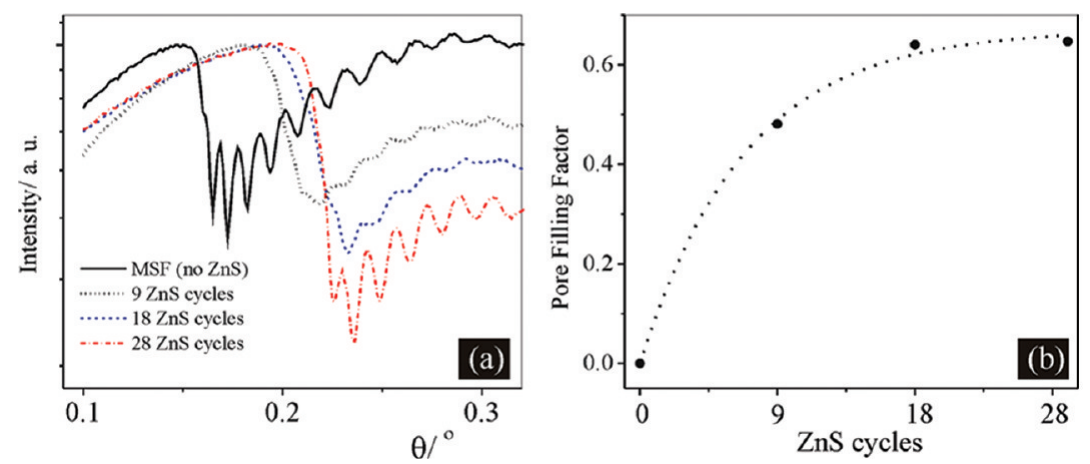

Figure 6. (a) Reflectivity data for the MSF prepared on ITO-covered glass substrate for different ZnS cycles and (b) pore filling factor with ZnS cycles. 
progressive increase of $\theta_{c}$ with each SILAR cycle, due to the higher electronic density of the MSF:ZnS films along infiltration of the sulfide into the MSF pores.

The $\mathrm{ZnS}$ volume fraction at each infiltration step $\left(F_{\mathrm{ZnS}}\right)$ can be calculated from the critical angle variation, according to eq 4

$$
F_{\mathrm{ZnS}}=\frac{\rho_{\mathrm{NC}}-\rho_{\mathrm{MSF}}}{\rho_{\mathrm{ZnS}}}
$$

where $\rho_{\mathrm{NC}}$ is the electronic density of the ZnS:MSF nanocomposite calculated from eq $3, \rho_{\mathrm{MSF}}$ is the electronic density of the empty exotemplate, and $\rho_{\mathrm{ZnS}}$ is the $\mathrm{ZnS}$ electronic density, which was calculated as $\rho_{\mathrm{ZnS}}=\delta_{\mathrm{ZnS}} n_{\mathrm{ZnS}} N_{\mathrm{A}} /$ $A_{\mathrm{ZnS}}$ where $\delta_{\mathrm{ZnS}}=4.09 \mathrm{~g} \mathrm{~cm}^{-3}$ is the $\mathrm{ZnS}$ mass density, $n_{\mathrm{ZnS}}$ is the $\mathrm{ZnS}$ atomic number, $A_{\mathrm{ZnS}}$ is the $\mathrm{ZnS}$ atomic mass, and $N_{\mathrm{A}}$ is Avogadro's number. The pore filling fraction (PFF) at each SILAR step can be calculated as shown in eq 5 .

$$
\mathrm{PFF}=\frac{F_{\mathrm{ZnS}}}{F_{\mathrm{MSF}}}
$$

Typical PFF values for 9, 18, and $28 \mathrm{ZnS}$ infiltration cycles are presented in Figure 6b, showing a plateau approximately at 20 $\mathrm{ZnS}$ infiltration cycles. This result corresponds to $60-65 \%$ of the pore relative volume, which is in good agreement with the results obtained from the UV/vis spectra. This limit of volume filling is similar to those found in nanoparticle-laden mesoporous systems, and might be due to partial blocking of the necks and pore clogging of the MSF as the number of infiltrations increases. ${ }^{25}$

Oscillations at angles higher than $\theta_{c}$ are due to the constructive interference of reflected X-rays on thin film interfaces and are known as XRR Kiessig fringes. ${ }^{30,40,44}$ In the higher angle region $\left(\theta>4 \theta_{c}\right)$ these periodic oscillations are related to the film thickness, $d$, according to

$$
d=\frac{\lambda}{2 \Delta(\sin \theta)}
$$

where $\lambda$ is the wavelength of the monochromatic X-rays used and $\Delta(\sin \theta)$ is the period of these oscillations. Interestingly, no changes in $\Delta(\sin \theta)$ are observed in any of the 4 cases shown in Figure 6a, leading to the conclusion that the $\mathrm{ZnS}$ does not deposit as a top layer, but that the semiconductor is indeed deposited within the mesopore system. In addition, Kiessig fringes with a significantly lower amplitude are observed in the case of those samples submitted to 9 and 18 infiltration cycles with respect to the pristine MSF and the nanocomposite film after 24 infiltration cycles. This amplitude decrease followed by an increase is due to the loss of electronic contrast between the film and the substrate along partial filling, and subsequent contrast increase due to the presence of a high volume fraction occupied by $\mathrm{ZnS}$, being consistent with the SAXS results of Figure $5 \mathrm{c}-\mathrm{e}$. In conclusion, a high $\mathrm{ZnS}$ loading of mesoporous silica thin films is achieved by the SILAR method reported here. An open issue is the $\mathrm{ZnS}$ growth mechanism, which can take place either by (a) heterogeneous $\mathrm{ZnS}$ nucleation on the pore surface in the first cycle followed by nanoparticle growth, (b) by successive nucleation-growth events on every cycle.

\section{c. Dissolution of ZnS:MSF: toward Mesoporous ZnS}

Thin Films. Successful nanocasting procedures must rely on compatible chemistries for synthesis and dissolution of the exotemplate matrix. Usually, $\mathrm{HF}$ aqueous solutions are the typical etching agents for $\mathrm{SiO}_{2}$; however, the $\mathrm{ZnS}$ structure might be damaged in the resulting low $\mathrm{pH}$ conditions. This issue can be circumvented by using ethanolic HF in order to assist $\mathrm{Si}(\mathrm{IV})$ solubilization while keeping low $\mathrm{ZnS}$ solubility. ${ }^{45,46,13}$ In this way, the $\mathrm{SiO}_{2}$ exotemplate can be successfully etched away, leaving behind an intact mesoporous $\mathrm{ZnS}$ structure when using a $0.5 \%$ ethanolic HF solution. However, due to the similar chemical behavior of the glass substrates and the silica matrix, it is necessary to provide a buffer layer between the glass substrate and the MSF:ZnS to prevent the detachment of the mesoporous $\mathrm{ZnS}$ layer during this etching process. In summary, the etching method was chosen in such a way that neither the ITO nor $\mathrm{ZrO}_{2}$ layers that covered the glass substrates nor the $\mathrm{ZnS}$ could be attacked by ethanolic HF.

Figure 7 shows four SEM images of MSF:ZnS samples after being exposed to the etching solution for different periods: 0,5 ,
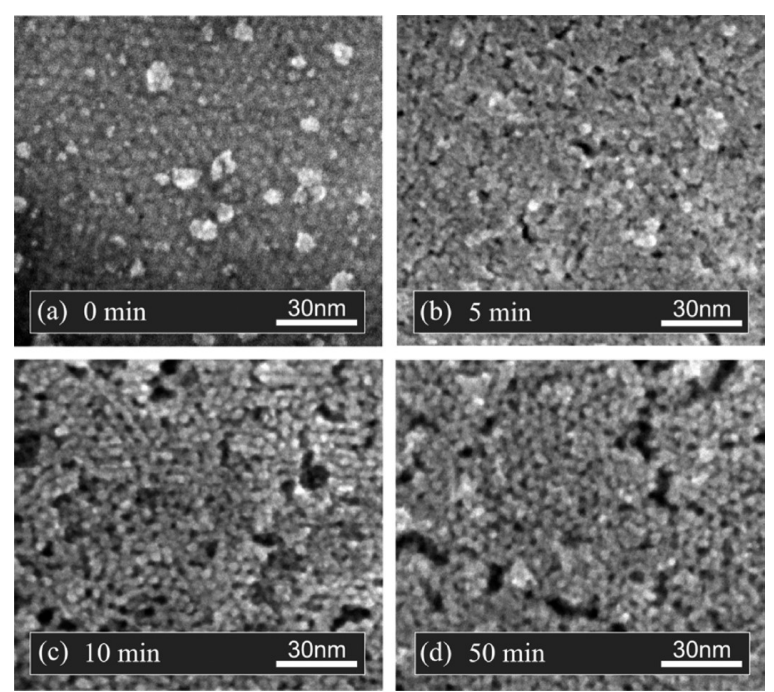

Figure 7. SEM images obtained from glass/ITO/SF:ZnS samples exposed to HF for (a) 0, (b) 5, (c) 10, and (d) $50 \mathrm{~min}$.

10, and $50 \mathrm{~min}$. Figure 7a corresponds to the MSF:ZnS thin film after 28 SILAR cycles and no etching process. After $5 \mathrm{~min}$ of exposure of the film to the HF solution, a partial dissolution of the $\mathrm{SiO}_{2}$ matrix can be observed (Figure 7b) while loosely surface bound $\mathrm{ZnS}$ aggregates are also washed away. This dissolution continues along etching time, as shown in Figure 7c, $\mathrm{d}$. The structure of the $\mathrm{ZnS}$ thin film remains unchanged to a large extent, and no detaching from the ITO covered glass substrate was observed neither by SEM nor visual inspection; indeed, ellipsometric porosimetry measurements (see below) were performed onto the $\mathrm{ZnS}$ films, confirming their integrity optical quality, thus their integrity.

The $\mathrm{ZnS}$ thin films etched for 0 and $50 \mathrm{~min}$, were analyzed with SEM-EDS to obtain information about the $\mathrm{SiO}_{2}$ content within the MSF: $\mathrm{ZnS}$ nanocomposite and the final mesoporous $\mathrm{ZnS}$ thin film. The corresponding SEM-EDS spectra, as well as the quantification of the elements are presented in Figure 8.

The nonetched MSF:ZnS film shows three strong EDS signals in the spectral maxima of $\mathrm{Si}, \mathrm{Zn}$ and $\mathrm{S}$, which correspond to the $\mathrm{SiO}_{2}$ matrix $(\mathrm{Si})$ and the $\mathrm{ZnS}$ infiltrated MSF (Zn and S), respectively. After 50 min etching with $0.5 \%$ ethanolic HF the Si peak in the SEM-EDS spectrum is hardly detectable, in contrast to the $\mathrm{Zn}$ and $\mathrm{S}$ signal, which are still present. This indicates that the $\mathrm{SiO}_{2}$ matrix is successfully removed by the etching step. Moreover, when the $\mathrm{SiO}_{2}$ framework is removed, a weak In peak arises from the ITO 

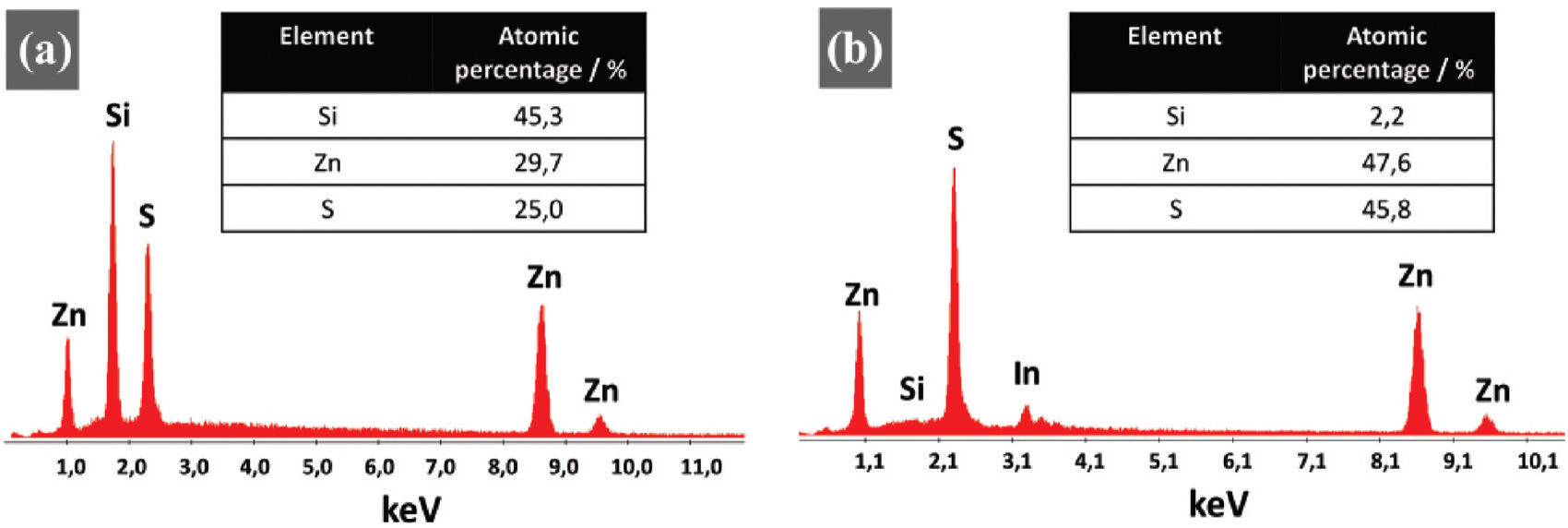

Figure 8. EDS spectra obtained from MSF:ZnS (28 infiltration cycles) exposed to ethanolic HF for (a) 0 and (b) 50 min. Samples were scraped off a glass/ITO substrate.
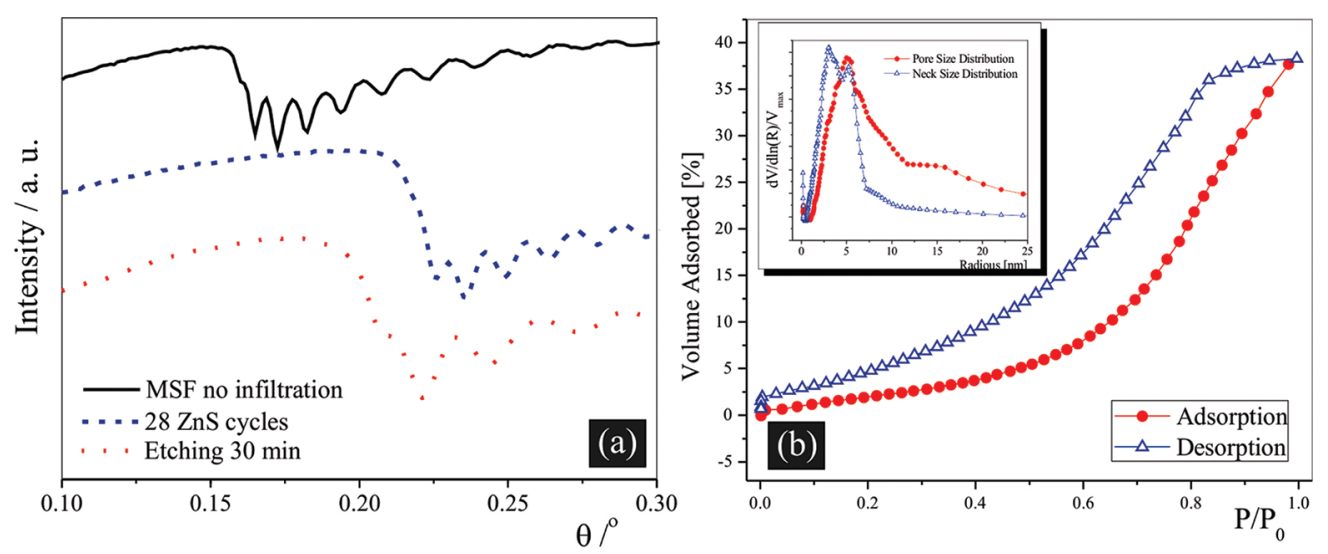

Figure 9. (a) XRR data for a not infiltrated MSF (black solid line), for the $28 \mathrm{ZnS}$ infiltration cycle (blue dashed line) and for an etched ZnS film (red dotted line), (b) Adsorption-desorption isotherms for a 30-min etched $\mathrm{ZnS}$ film. Inset: pore and neck distribution.

layer of the glass/ITO substrate (Figure $8 \mathrm{~b}$ ); this is due to the lower density of the resulting porous $\mathrm{ZnS}$ film. In both cases, untreated and etched MSF:ZnS, the ratio between $\mathrm{Zn}$ and $\mathrm{S}$ is approximately $1: 1$, which corresponds well to the formation of ZnS.

In order to quantify the porosity of the $\mathrm{ZnS}$ mesoporous film after the etching process, the XRR technique was employed. Figure 9a shows the variation of the critical angle for the three main stages in the whole process. The black solid line shows a small $\theta_{c}$ for the not infiltrated MSF while an important increment of the $\theta_{c}$ is observed for the 28-cycle $\mathrm{ZnS}$ infiltrated film (blue dashed line), reflecting the increase in the electronic density of the nanocomposite, as already shown in Figure 6. After etching the $\mathrm{SiO}_{2}$ matrix, a pronounced shift to a lower critical angle is observed, indicating that the electronic film density has decreased to a value between the empty MSF and the full MSF:ZnS, due to the removal of the $\mathrm{SiO}_{2}$ exotemplate. As there are no representative changes in $\theta_{c}$ after $30 \mathrm{~min}$, and considering the results obtained in the SEM-EDS measurements, it is possible to assume that almost all $\mathrm{SiO}_{2}$ has been removed. Consequently, a $\mathrm{ZnS}$ mesoporous thin film has been obtained with an overall accessible porosity of approximately $45 \%$ calculated from XRR measurements (compared to dense $\mathrm{ZnS})$.

Figure $9 \mathrm{~b}$ shows the adsorption and desorption curves obtained by EEP for a $\mathrm{ZnS}$ film derived from $30 \mathrm{~min}$ etching of the silica film over ITO. An adsorbed volume of $38 \%$ is registered by this technique, in good agreement with the $45 \%$ obtained by XRR. The inset in Figure $9 \mathrm{~b}$ shows the pore and neck size distribution obtained for this $\mathrm{ZnS}$ film. The corresponding isotherms show rather large pore size distributions, which correspond to different pore sizes, suggesting that the available porosity due to the removed $\mathrm{SiO}_{2}$ generates different kind of channels in the film, as clearly showed in Figure 7 . The pore size range presents relatively well-defined maxima at around 6 and $15 \mathrm{~nm}$. From the data obtained from EEP technique it is also possible to determine that the film thickness decreases to $80 \mathrm{~nm}$ after etching, in agreement with the Kiessig fringes observable in Figure 9a, where the peak separation of the $30 \mathrm{~min}$ etched $\mathrm{ZnS}$ film (red dotted line) approximately doubles the one of the original film (black solid line). The incomplete filling of the original silica exotemplate (ca. $60 \%$ of the pore volume) leads to an open $\mathrm{ZnS}$ framework which is not totally connected. Upon etching of the silica exotemplate, the $\mathrm{ZnS}$ backbone can either reorganize or partially deteriorate, leading to a final thinner film. Efforts are being directed to address this issue in detail and will be communicated in a further publication. However, it must be stressed that the $\mathrm{ZnS}$ films obtained present accessible mesoporosity, optical homogeneity, and good adhesion to the ITO substrate.

Another important information obtained from EEP technique is the refractive index of mesoporous $\mathrm{ZnS}$. EEP measurements show that under dry atmosphere $(0 \% \mathrm{RH}), n_{630}$ 
$=1.57$, increasing up to 1.74 when the material is exposed to $100 \%$ humidity. This significant increment of $\Delta n=0.17$ implies that the film obtained is of potential interest for optical sensing applications. $^{47}$

To obtain further structural information about the mesoporous $\mathrm{ZnS}$, the thin film was studied by TEM and selected area electron diffraction. Figure 10a shows a TEM
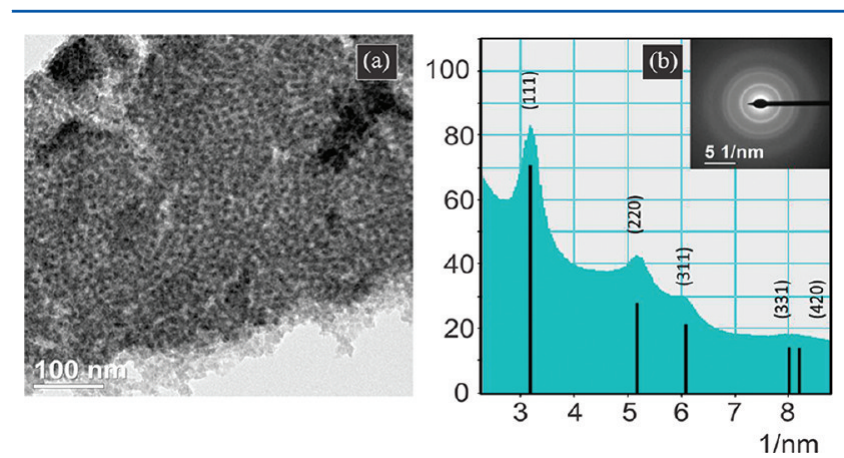

Figure 10. (a) TEM image and (b) electron diffraction analysis and diffraction rings of a mesoporous $\mathrm{ZnS}$ thin film scratched off a glass/ ITO substrate after removal of the $\mathrm{SiO}_{2}$ matrix. Black lines give the position of the peaks according to the powder diffraction file (PDF) 05-05665 of the International Centre for Diffraction Data for sphalerite type $\mathrm{ZnS}$.

image recorded from a mesoporous $\mathrm{ZnS}$ thin film scratched from a glass/ITO substrate, obtained after etching in ethanolic $\mathrm{HF}$ solution for $50 \mathrm{~min}$. The $\mathrm{ZnS}$ pores show a long-range order, which is consistent with recorded SEM images of the MSF:ZnS films. SAED analysis was evaluated by using the method described by Mitchell (Figure 10b). ${ }^{48}$ The electron diffraction pattern shows five well resolved main rings, corresponding to the diffraction of different planes of the randomly ordered nanocrystallites. The positions of the rings can be assigned to sphalerite type $\mathrm{ZnS}$, which is in accordance with the Powder Diffraction File (PDF) 05-05665. In this way, a robust mesoporous nanocrystalline $\mathrm{ZnS}$ can be deposited onto a semiconductor substrate. Moreover, the mesoporous $\mathrm{ZnS}$ thin film reported here shows photoluminescence (see the Supporting Information); further studies are under way to delve with these properties.

\section{CONCLUSIONS}

In this contribution, we present the successful preparation of organized mesoporous $\mathrm{ZnS}$ thin layers using a nanocasting approach. The $\mathrm{SiO}_{2}$ exotemplate prepared by evaporation induced self-assembly process using an F-127 polymeric template is highly accessible and thus acts as an adequate exotemplating agent, facilitating the infiltration of the MSF matrix with $\mathrm{ZnS}$. The SILAR route proves to be a useful approach to build nonoxide mesoporous nanophases, due to the high control of the ion infiltration-surface precipitation processes that are mainly confined in the pore interior. This strategy relies on the control of several phenomena taking place in the mesoporous exotemplate material upon loading of the precursors of the desired phase, and the further confined growth of said phase. In the first place, pore charge, size and interconnectivity are extremely important, for they determine the affluence and organized deposition of the precursors; these effects are key for example in perm-selectivity, or controlled transport. ${ }^{49}$ Indeed, the controlled synthesis presented here demonstrates that these confinement-dependent properties (a central feature of mesoporous systems) can be exploited as a synthetic tool. ${ }^{50}$ In the subsequent filling step, the exotemplate removed by exposing the film to an ethanolic HF solution yields a stable and nearly $\mathrm{SiO}_{2}$-free mesoporous $\mathrm{ZnS}$ thin film on a transparent electrode such as a glass/ITO substrate. The sequential strategy presented here can be extended in principle to more complex materials, in particular to sulfides or selenides, such as $\mathrm{Cu}(\mathrm{In}, \mathrm{Ga}) \mathrm{Se}_{2}$ (CIGS) or related phases that present high interest in solar cells. The combination of these robust and highly accessible nanostructured materials with metals or conductive polymers ${ }^{51}$ can lead to new developments for sensors or heterogeneous junctions used in inorganic-organic hybrid photovoltaics.

\section{ASSOCIATED CONTENT}

\section{Supporting Information}

Photoluminescence spectra of mesoporous $\mathrm{ZnS}$ thin films is provided. This material is available free of charge via the Internet at http://pubs.acs.org/.

\section{AUTHOR INFORMATION}

\section{Corresponding Author}

*E-mail: gsoler@cnea.gov.ar. Tel.: 005491165071916.

\section{Notes}

The authors declare no competing financial interest.

\section{ACKNOWLEDGMENTS}

The authors thank the Christian Doppler Research Association (CDG), the federal ministry of Economy, Family and Youth of Austria, ANPCyT (PICT 1848, PAE 2006-37063) and CONICET (PIP 11220100100186) for financial support. ABTLus is kindly acknowledged for permitting access to LNLS synchrotron (projects XRD2-10041, XRD2-11042, SAXS1-11060). A.F. acknowledges a Marietta Blau Stipendium for collaborative exchange. G.S.-I., M.L.M.-R., and A.W. are members of CONICET and Gabbos (RG \#1-FD06). Violeta Cardinaux is kindly acknowledged for postponing her birth date, leaving time for sending the corrected version.

\section{REFERENCES}

(1) Kresge, C. T.; Leonowicz, M. E.; Roth, W. J.; Vartuli, J. C.; Beck, J. S. Nature 1992, 359, 710-712.

(2) Yanagisawa, T.; Shimizu, T.; Kuroda, K.; Kato, C. Bull. Chem. Soc. Jpn. 1990, 63, 988-992.

(3) Ding, Y.; Chen, M.; Erlebacher, J. J. Am. Chem. Soc. 2004, 126, 6876-6877.

(4) Hu, J. S.; Ren, L. L.; Guo, Y. G.; Liang, H. P.; Cao, A. M.; Wan, L. J.; Bai, C. L. Angew. Chem., Int. Ed. 2005, 44, 1269-1273.

(5) Reddy, K. M.; Satyanarayana, L.; Manorama, S. V.; Misra, R. D. K. Mater. Res. Bull. 2004, 39, 1491-1498.

(6) Li, J.; Kessler, H.; Soular, M.; Khouchaf, L.; Tuilier, M.-H. Adv. Mater. 1998, 10, 946-949.

(7) Nistor, L. C.; Mateescu, C. D.; Birjega, R.; Nistor, S. V. Appl. Phys. A: Mater. Sci. Process. 2008, 92, 295-301.

(8) Braun, P. V.; Osenar, P.; Tohver, V.; Kennedy, S. B.; Stupp, S. I. J. Am. Chem. Soc. 1999, 121, 7302-7309.

(9) Rana, R. K.; Zhang, L.; Yu, J. C.; Mastai, Y.; Gedanken, A. Langmuir 2003, 19, 5904-5911.

(10) Braun, P. V.; Osenar, P.; Twardowski, M.; Tew, G. N.; Stupp, S. I. Adv. Funct. Mater. 2005, 15, 1745-1750.

(11) Van Gough, D.; Wolosiuk, A.; Braun, P. V. Nano Lett. 2009, 9, 1994-1998. 
(12) Wolosiuk, A.; Armagan, O.; Braun, P. V. J. Am. Chem. Soc. 2005, 127, 16356-16357.

(13) Son, D.; Wolosiuk, A.; Braun, P. V. Chem. Mater. 2009, 21, 628-634.

(14) Schüth, F. Chem. Mater. 2001, 13, 3184-3195.

(15) Karakaya, C.; Türker, Y.; Albayrak, C.; Dag, O. Chem. Mater. 2011, 23, 3062-3071.

(16) Türker, Y.; Karakaya, C.; Dag, Ö. Chem.-Eur. J. 2012, 18, 3695-3705.

(17) Lepoutre, S.; Julián-López, B.; Sanchez, C.; Amenitsch, H.; Linden, M.; Grosso, D. J. Mater. Chem. 2010, 20, 537-542.

(18) Yang, H.; Zhao, D. J. Mater. Chem. 2005, 15, 1217-1231.

(19) Flath, J.; Meldrum, F. C.; Knoll, W. Thin Solid Films 1998, 327$329,506-509$.

(20) Kosmulski, M. Chemical Properties of Material Surfaces; CRC: Boca Raton, FL, 2001; Vol. 102.

(21) Tiemann, M. Chem.-Eur. J. 2007, 13, 8376-8388.

(22) Waitz, T.; Wagner, T.; Sauerwald, T.; Kohl, C. D.; Tiemann, M. Adv. Funct. Mater. 2009, 19, 653-661.

(23) Valdés-Solís, T.; Fuertes, A. B. Mater. Res. Bull. 2006, 41, 21872197.

(24) Schüth, F. Angew. Chem., Int. Ed. 2003, 42, 3604-3622.

(25) Lepoutre, S.; Smått, J.-H.; Laberty, C.; Amenitsch, H.; Grosso, D.; Lindén, M. Microporous Mesoporous Mater. 2009, 123, 185-192.

(26) Grosso, D.; Cagnol, F.; Soler-Illia, G. J. D. A. A.; Crepaldi, E. L.; Amenitsch, H.; Brunet-Bruneau, A.; Bourgeois, A.; Sanchez, C. Adv. Funct. Mater. 2004, 14, 309-322.

(27) Nicolau, Y. F. Appl. Surf. Sci. 1985, 22-23, 1061-1074.

(28) Niesen, T. P.; De Guire, M. R. Solid State Ionics 2002, 151, 6168.

(29) Ristov, M.; Sinadinovski, G.; Grozdanov, I.; Mitreski, M. Thin Solid Films 1989, 173, 53-58.

(30) van der Lee, A. Solid State Sci. 2000, 2, 257-278.

(31) Angelome, P. C.; Fuertes, M. C.; Soler-Illia, G. Adv. Mater. 2006, 18, 2397-+.

(32) Bass, M.; Mahajan, V. N.; Van Stryland, E. Handbook of Optics: Design, Fabrication, and Testing; Sources and Detectors; Radiometry and Photometry; McGraw-Hill Professional: New York, 2009; Vol. 4.

(33) Synowicki, R. A. Thin Solid Films 1998, 313-314, 394-397.

(34) Boissiere, C.; Grosso, D.; Lepoutre, S.; Nicole, L.; Bruneau, A. B.; Sanchez, C. Langmuir 2005, 21, 12362-12371.

(35) Kruk, M.; Jaroniec, M. Chem. Mater. 2003, 15, 2942.

(36) Lowell, S.; Shields, J. E.; Thomas, M. A.; Thommes, M. Characterization of Porous Solids and Powders: Surface Area, Porosity and Density; Springer: New York, 2006.

(37) Galarneau, A.; Desplantier, D.; Dutartre, R.; Di Renzo, F. Microporous Mesoporous Mater. 1999, 27, 297-308.

(38) Sakatani, Y.; Grosso, D.; Nicole, L.; Boissière, C.; Soler-Illia, G.

J. D. A. A.; Sanchez, C. J. Mater. Chem. 2006, 16, 77-82.

(39) Brunsen, A.; Calvo, A.; Williams, F. J.; Soler-Illia, G. J. A. A.; Azzaroni, O. Langmuir 2011, 27, 4328-4333.

(40) Fuertes, M. C.; Marchena, M.; Marchi, M. C.; Wolosiuk, A.; Soler-Illia, G. Small 2009, 5, 272-280.

(41) Nicolau, Y. F.; Dupuy, M.; Brunel, M. J. Electrochem. Soc. 1990, 137, 2915-2924.

(42) Rossetti, R.; Hull, R.; Gibson, J. M.; Brus, L. E. J. Chem. Phys. 1985, 82, 552-559.

(43) Hodes, G. Chemical Solution Deposition of Semiconductor Films;

CRC Press: Boca Raton, FL, 2003.

(44) Gibaud, A.; Hazra, S. Curr. Sci. 2000, 78, 1467-1477.

(45) Ràfols, C.; Herodes, K.; Beltrán, J. L.; Bosch, E.; Rosés, M. J. Electroanal. Chem. 1997, 433, 77-83.

(46) Velikov, K. P.; Van Blaaderen, A. Langmuir 2001, 17, 47794786.

(47) Fuertes, M. C.; López-Alcaraz, F. J.; Marchi, M. C.; Troiani, H. E.; Luca, V.; Míguez, H.; Soler-Illia, G. J. D. A. A. Adv. Funct. Mater. 2007, 17, 1247-1254.

(48) Mitchell, D. R. G. Ultramicroscopy 2008, 108, 367-374.
(49) Schoch, R. B.; Han, J.; Renaud, P. Rev. Mod. Phys. 2008, 80, 839-883.

(50) Calvo, A.; Fuertes, M. C.; Yameen, B.; Williams, F. J.; Azzaroni, O.; Soler-Illia, G. J. A. A. Langmuir 2010, 26, 5559-5567.

(51) Soler-Illia, G. J. A. A.; Azzaroni, O. Chem. Soc. Rev. 2011, 40, $1107-1150$. 